\title{
RAFS: A computer-assisted robotic system for minimally invasive joint fracture surgery, based on pre- and intra-operative imaging
}

\author{
G. Dagnino ${ }^{1}$; I. Georgilas ${ }^{1}$; S. Morad ${ }^{1}$; P. Gibbons ${ }^{1}$; P. Tarassoli ; R. Atkins ${ }^{2}$; S. Dogramadzi ${ }^{1}$ \\ ${ }^{1}$ Bristol Robotics Laboratory, Coldharbour Lane, Frenchay, BS16 1QY, Bristol, United Kingdom \\ ${ }^{2}$ University Hospitals Bristol, United Kingdom
}

\section{Abstract}

The integration of minimally invasive robotic assistance and image-guidance can have positive impact on joint fracture surgery, providing a better clinical outcome with respect to the current open procedure. In this paper, a new design of the RAFS surgical system is presented. The redesign of the robotic system and its integration with a novel 3D navigation system through a new clinical workflow, overcomes the drawbacks of the earlier prototype. This makes the RAFS surgical system more suitable to clinical scenarios in the operating theatre. System accuracy and effectiveness are successfully demonstrated through laboratory trials and preliminary cadaveric trials. The experimental results demonstrate that the system allows the surgeon to reduce a 2 -fragment distal femur fracture in a cadaveric specimen, with a reduction accuracy of up to $0.85 \mathrm{~mm}$ and $2.2^{\circ}$. Preliminary cadaveric trials also provided a positive and favorable outcome pointing to the usability and safety of the RAFS system in the operating theatre, potentially enhancing the capacity of joint fracture surgeries.

\section{Introduction}

State-of-the-art surgical treatment of lower limb joint fractures involves an open incision into the knee to expose the fractured bones and to allow the surgeon to perform the anatomical reduction of the fracture. This consists in manually repositioning the broken bone fragments to reconstruct the fractured bone as precisely as possible, ensuring the correct joint functionality and avoiding postoperative symptoms such as arthritis, chronic pain, and (partial) disablement [1]. Anatomical reduction is followed by fracture fixation using a metallic plate and screws or intramedullary nails [2]. Although the effectiveness of the open reduction and internal fixation (ORIF) technique has been demonstrated in practice, it is associated with extensive soft tissue damage, higher risk of infection, longer hospitalization and rehabilitation time, and high health-related costs [3]. Minimally invasive techniques can potentially reduce the said problems by allowing the surgeon to manipulate the fracture fragments through small incisions in the flesh. The actual pose of the fragments is estimated intra-operatively by two orthogonal fluoroscopic images in the coronal and sagittal planes. However, 2D images with a limited field of view and low resolution do not provide enough information to the surgeon in respect of the fracture alignment and rotation, possibly causing a misinterpretation of the corrective parameters. In fact, this is a 3D problem which requires restoring six parameters-three translations and three rotations-to achieve an optimal anatomical reduction and pose correction of 
the articular surface. Moreover, the high forces occurring during the reduction process increase the physical load on the surgeon preventing the reduction movements and occasionally resulting in suboptimal fracture reduction [4]. Integration of robotic assistance and image guidance can potentially have a positive impact in overcoming the issues identified above, through increased reduction accuracy and enhanced pre- and intraoperative 3D imaging. Several surgical systems which integrate robotic assistance and 3D image guidance are reported in literature [5]-[6][7][8]. The application of these systems is restricted to long bone fractures, which usually have a smaller number of larger fragments that can be managed using the current clinical setup (2D fluoroscopic imaging). Joint fractures typically require higher reduction accuracy to restore the articular surface [3] (which is a 3D problem) and therefore are more difficult to solve using 2D imaging. Earlier research by the authors of this paper resulted in a surgical prototype that can successfully accomplish the reduction of 1-fragment distal femur fracture on phantom models [9], [10]. However, within the ultimate aim to move the system into a clinical scenario, key issues were identified related to: the fracture types that can be reduced using the RAFS (Robot-Assisted Fracture Surgery) system (only 1 fragment at the time can be manipulated by the robot), traction can't be applied through the tibia to help with the reduction, and a non-optimal clinical workflow. All these pose serious restrictions to the system's applicability in the real surgical environment. This paper describes a new RAFS system design (Fig. 1), its integration into the operating environment through new navigation system and clinical workflow, and its evaluation through laboratory experiments and a pilot study on cadavers, improving the limitations of the earlier prototype.

\section{Clinical Requirements}

Two different types of 2-fragment distal femur fractures (DFFs) have been considered for the development and testing of the RAFS system, namely articular Y-shape 33-C1 and articular T-shape 33-C1 fractures (Fig. 2) [2]. The main criterion for this selection was the large size of the fragments (medial and lateral condyles) allowing for the secure insertion of orthopedic manipulation pins through small incisions (percutaneous manipulation). These kind of fractures usually require open surgery involving manual traction of the tibia to restore the appropriate length and rotation of the joint and manual manipulation of the fragments [2], [3]. The required reduction accuracy has been set to $1 \mathrm{~mm}$ (translational) and $5^{\circ}$ (rotational) [3], [9]. The required traction capacity (350N) for the system has been defined by measuring forces applied by the surgeons during in-vivo lower limb surgical procedures as described in [11]. The load required for manipulating the fragments $(100 \mathrm{~N}, 10 \mathrm{Nm})$ has been defined by measuring forces and torques applied by the surgeons while reducing distal femur fractures (two T-shape fractures and two Y-shape fractures) on four cadaveric specimens. Fragments were manipulated through instrumented [11] orthopedic pins inserted into them through small incisions.

\section{Limitations with Earlier RAFS Prototype}

The earlier RAFS surgical system prototype presented in [9] was created to provide improved accuracy and safety for minimally invasive intra-articular fracture surgeries, through a redesigned robotic architecture, improved control strategy, and real-time 3D imaging. Validation trials on bone models proved a fracture reduction accuracy of only $1.15 \mathrm{~mm}$ and $1.3^{\circ}$. However, these trials exposed also some issues that might restrict the system's applicability to a real surgical environment: 1 ) Manipulating fracture fragments: with this earlier prototype, only 1 fragment could be manipulated. Although DFFs can have only one fragment, complex DFFs are multi-fragmented and require higher surgical skills to be reduced (e.g. 33-C1) [2], [3]. Therefore, the RAFS system should be able to manipulate at least 2 fragments at the same time to be used in a real clinical procedure. 2) Applying 
traction through the tibia: multi-fragmented fractures usually require manual traction of the tibia to restore the appropriate length and rotation of the joint [3]. This also creates the required space inside the joint to manipulate the fragments while minimizing the load applied. 3) Improving the clinical workflow: the clinical workflow for the reduction of joint fractures using the earlier RAFS prototype is presented in [9]. The pre-operative part of this clinical workflow consists in the insertion of the orthopedic pins in the fragments, followed by the CT scan of the fracture and the creation of 3D models of the bone fragments, which enables intra-operative 3D image guidance [9]. This workflow presents two main issues: 1 ) the pins are inserted into the bones outside the operating theatre, which is not realistic since this is a surgical procedure; 2 ) the pins should be inserted into the fragments after getting the CT scan of the fracture, because presence of metal pins creates artifacts in the CT images that can affect the accuracy of the models, and, therefore, the system's image guidance. This requires a redesign of the navigation system and a new clinical workflow.

\section{RAFS System Design}

The new RAFS system should be able to: i) manipulate 2 bone fragments simultaneously; and ii) apply traction force to the joint through the tibia. The new elements of the RAFS system are (Fig. 1): 1) Robotic Fracture Manipulator (RFM) and Carrier Platform (CP): a second RPM-a computercontrolled 6-DOF parallel-robot with 6-DOF load cell [9]-is included in the system to manipulate a second bone fragment $\left(0.03 \pm 0.01 \mathrm{~mm}\right.$ translational accuracy and $0.12 \pm 0.01^{\circ}$ rotational accuracy [12]) connected through a custom-designed manipulation pin (described below). The RPM is mounted on a second CP (4-DOF, computer-controlled) which is used for the coarse positioning of the RPM close to the manipulation pin as described in [9]. Two RFM-CP systems allow the manipulation of two fragments at the same time. The kinematics and control of the RPM and the CP are described in [9]. 2) External Robot (ER): this is a computer-controlled version of a traction table, i.e. an operating table which allows the application of a constant and adjustable traction force to the lower limb through the tibia [11]. The ER is a 4-DOF mechanism (see Fig. 8a), connected to the tibia through an orthopedic boot (Fig. 1 and Fig. 6c). A force sensor is mounted between the ER and the boot enabling force control. The ER allows the manipulation of the tibia to restore the appropriate length and rotation of the joint. The kinematics and control of the ER are described in the Appendix.

The new elements were integrated into the RAFS surgical system. The final prototype (Fig. 1) includes also a system workstation to operate the robot (see [9]), and a navigation system consisting of reduction software, optical tracking system (Polaris Spectra, NDI Inc.), and contact-less user controller (Leap Motion). The navigation system allows the surgeon to interact with CT-generated 3D models of the fracture using the user controller for pre-operative planning of fracture reduction, i.e. virtually reduces the fracture [10]. It also provides real-time update of the 3D models through the optical tools placed on the orthopedic pins inserted into the bone fragments (see below). Accuracies of the RAFS system sub-components are reported in [9], [10], [12].

\section{Navigation System and Clinical Workflow}

The navigation system allows the surgeon to pre-plan the surgery before entering the operating theatre, i.e. virtual reduction. Real-time intra-operative image-guidance is provided to help the surgeon to physically reduce the fracture using the robotic system. The navigation system and the clinical workflow have been improved (with respect to [9], [10]) to allow full pre-operative planning and to fix the issues of the earlier version (see Section III), i.e. to be used in a real clinical environment. Two-fragment DFFs (i.e. Y-shape 33-C1 and T-shape 33-C1 Fig. 2) have been used to design the navigation system and the new clinical workflow. 


\section{A. Pre-Operative Planning}

3D models (STL format) of each bone fragment are generated by the segmentation of pre-operative CT data of the fracture. The surgeon virtually reduces the fracture using the GUI (see Fig. 6a) by manipulating the models, i.e. F1 and F2 to match FEM (which remains fixed). This generates the desired pose of fragments F1 (FIPd) and F2 (F2Pd) with respect to the femur, in order to achieve the anatomical reduction of the fracture. Pre-operative reduction results are then used intra-operatively to calculate the motion commands for the robot to physically reduce the fracture as described below.

\section{B. Intra-Operative 2D/3D Registration}

In the operating theatre, the femur is physically fixed to the operating table using Taylor Spatial Frame and k-wires. 4 orthopedic pins $\left(\mathrm{OP}_{\mathrm{s}}\right)$ are inserted into the bone fragments (Fig. 3a). Two $\mathrm{OP}_{\mathrm{s}}$ are inserted into the fragments to be manipulated by the robot: i.e. $\mathrm{OP}_{\mathrm{F} 1}$ is inserted in $\mathrm{F}_{1}$ and $\mathrm{OP}_{\mathrm{F} 2} \mathrm{in} \mathrm{F} 2$. Two further $\mathrm{OP}_{\mathrm{s}}$ are inserted into the tibia $\left(\mathrm{OP}_{\mathrm{T} 1}\right)$ and the femur $\left(\mathrm{OP}_{\mathrm{FEM}}\right) . \mathrm{OP}_{\mathrm{s}}$ present two sections: a threaded section ( $\mathrm{M} 4 \times 1$ to be screwed into the bone) and a tool section (one-flat-face geometry), whose objective is supporting registration tools and optical tools (Fig. 4) (see below). The relative position of each pin with respect to the bone fragment in which it is inserted needs to be calculated. Custom-designed registration tools (RTs) have been 3D-printed (Fig. 5a). Each registration tool is made of Acrylonitrile-Butadiene-Styrene (ADP) (radiolucent) and contains three stainless steel beads (radiopaque). RTs have been designed to be rigidly connected and locked (using a locking screw) in a unique way into $\mathrm{OP}_{\mathrm{s}}$ through the tool section (Fig. 4b). Two fluoroscopic images from different angles (i.e. $90^{\circ}, 30^{\circ}$ ) of each fragment-pin-registration tool are taken (e.g. Fig. 5b) and imported in the reduction software, together with the CAD 3D models of the registration tools placed on the pins and the 3D models of the fracture generated by pre-operative $C T$ dataset. The $2 \mathrm{D} / 3 \mathrm{D}$ registration (Fig. $5 \mathrm{C}$ ) starts with the estimation of relative pose between the 2 fluoroscopic images and the registration tool using bead-based registration algorithm [13] (i.e. using the beads shown in Fig. 5b, c). Then, the 3D model of the bone fragment (i.e. the red model in Fig. 5c) is registered with the fluoroscopic images. Initial manual registration (coarse registration) is required to speed up the following optimization step: the contour of the bone fragment is segmented on the two fluoroscopic images, and a spline-based registration method [14] is applied to minimize the distance between the projected 3D contour of the model and the segmented 2D contour on each fluoroscopic image (i.e. fuchsia and yellow contours in Fig. 5c). Once the registration is completed, the relative pose between each pin and its fragment is known, and the homogeneous transformations PiTFi can be calculated (Fig. 5d). PiTFi are considered to be constant during the operation.

\section{Physical Reduction}

Registration tools are replaced by optical tools (Fig. 3b): optical tool $\mathrm{OT}_{\mathrm{Tl}}$ is placed on the pin $\left(\mathrm{OP}_{\mathrm{T} 1}\right)$ inserted in the tibia ( $\mathrm{TI}), \mathrm{OT}_{\mathrm{FEM}}$ on the pin $\left(\mathrm{OP}_{\mathrm{FEM}}\right)$ inserted in the femur $(\mathrm{FEM}), \mathrm{OT}_{\mathrm{FI}}$ on the pin $\left(\mathrm{OP}_{\mathrm{FI}}\right)$ fragment $1(\mathrm{~F} 1)$, and $\mathrm{OT}_{\mathrm{F} 2}$ on the pin $\left(\mathrm{OP}_{\mathrm{F} 2}\right)$ inserted in fragment 2 (F2). Two optical tools are also placed on each RFM (i.e. $\mathrm{OT}_{\mathrm{RFM} 1}$ and $\mathrm{OT}_{\mathrm{RFM} 2}$ ) enabling vision-based closed-loop control [12]. As mentioned previously, the orthopedic pins were designed to be connected in a unique way to the optical tools (through the tool section), having their coordinate frames coincident, i.e. CFPi=CFOTi Fig. 4c. $\mathrm{OT}_{\mathrm{s}}$ have locking screws that ensure their perfect alignment with the pins. The optical tracker provides the actual pose of each fragment (F1, F2, TI, FEM) by tracking its pin, enabling the intraoperative real-time image guidance. A full description of the coordinate frames and transformation involved is provided in [10]. A surgeon's assistant connects the ER to the patient's foot, RFM1 to the OPF1, and RFM2 to the OPF2. The surgeon applies the desired traction to the joint through the external robot with the help of the intra-operative image-guidance. Results of the pre-operative planning F1Pd and F2Pd i.e. the virtual reduction parameters, are uploaded into the intra-operative 
procedure, and the corresponding desired pose in the task space for the RFMs RFMIPd and RFM2Pd are calculated as described in [10] to achieve the fracture reduction. The surgeon perform intra-operative virtual reduction to generate desired trajectories for the RFMs, e.g. to deal with tiny bone fragments and tissue that might interfere with the reduction. The RFMs autonomously execute the desired movements for F1 and F2-based on the surgeon's pre- and intraoperative planning (virtual reduction)-to achieve the physical reduction of the fracture, while FEM remain fixed. The intra-operative imaging updates the actual pose of the fragments in real time, and the surgeon can check the reduction in 3D without the use of any other intra-operative imaging device. If the reduction is acceptable, then the surgeon proceeds with the fixation of the fracture by using plate and screws or intramedullary nail, and the surgery ends.

\section{Experimental Validation}

The RAFS surgical system has been tested through laboratory trials on bone models and cadaveric trials. A brief video showing the experiments and the clinical workflow accompanies this manuscript. All CT images were acquired with a SOMATOM Sensation 16 (Siemens Healthcare) CT scanner with a voxel size of $0.58 \mathrm{~mm} \times 0.58 \mathrm{~mm} \times 0.75 \mathrm{~mm}$. Intra-operative fluoroscopic images were taken with an OEC Fluorostar (GE) C-arm. The metrics chosen for the system evaluation were the root-mean-squarederror (RMSE), the maximum absolute error (MAE), and the load applied by the robot (RE and RFMs) measured during the physical reductions. The surgical procedure time (excluding the preoperative planning time) has also been measured during the cadaveric trials.

\section{A. Laboratory Trials}

Five reductions of a Y-shape 33-C1 DFF and 5 reductions of a T-shape 33-C1 DFF were performed following the new clinical workflow. The setup is shown in Fig. 1. A leg model has been manufactured including solid-foam femur and tibia encased in semi-flexible foam simulating the skin and the soft tissue surrounding the joint (i.e. muscles and flesh). Polypropylene bands were connected between the distal part of the femur and the proximal part of the tibia, in order to simulate knee ligaments (Fig. 3). $\mathrm{OP}_{\mathrm{Fl}}, \mathrm{OP}_{\mathrm{F} 2}$, and $\mathrm{OP}_{\mathrm{FEM}}$ were inserted in the un-fractured bone models and their relative poses (FIPgoal and F2Pgoal) were provided by the optical tracker to obtain a ground truth for the reduction assessment. The bone models were then fractured keeping $\mathrm{OP}_{\mathrm{Fl}}$ in $\mathrm{F} 1, \mathrm{OP}_{\mathrm{F} 2}$ in $\mathrm{F} 2$ and OPFEM in FEM (Fig. 3a) and CT scanned. The proposed clinical workflow was followed to reduce each fracture. The average registration accuracy was $0.74 \pm 0.04 \mathrm{~mm}$ (mTRE). After the physical reduction, the actual pose of $\mathrm{FI}(\mathrm{FIPa})$ and $\mathrm{F} 2$ (F2Pa)was measured by the optical tracker, allowing for the physical reduction accuracy assessment, as the translational and rotational error between the ground truth poses FIPgoal and F2Pgoal and the actual poses FIPa and F2Pa [10]. Experimental results are reported in Table I.

\section{B. Cadaveric Trials}

Two cadaver specimens (two right lower limbs from males) were used to evaluate the entire method as follows. An orthopedic surgeon fractured the distal part of each femur creating a Y-shape 33-C1 fracture in one leg and a T-shape 33-C1 in the other leg. This process, called osteotomy, was carried out using drill and osteotome through a short supra-patellar incision of about $3 \mathrm{~cm}$. Again, the clinical workflow has been applied to each specimen. The setup is shown in Fig. 6 . The average registration accuracy was $1.15 \pm 0.35 \mathrm{~mm}$ (mTRE). After each physical reduction, the surgeon temporarily fixated the fracture using a set of $\mathrm{k}$-wires to ensure the immobilization of the fragments during the assessment of the reduction. The fragments were kept in the reduced position by the RFMs during the fixation, thus the reduction assessment was not affected by the fixation. After fixating the fracture, the surgeon assessed the reduction with the aid of fluoroscopic images [2]. Fluoroscopic images of the 
reduced fracture were taken: one in the coronal plane, one in the sagittal-lateral plane, and one in the sagittal-medial plane. The assessment of reduction accuracy was completed post hoc using the fluoroscopic images and the tools available in the Sante DICOM Viewer (Santesoft). Reduction is defined as the restoration of the normal anatomical alignment. In this regard, the surgeon measured the translational and rotational deviations on the fluoroscopic images as shown in Fig. 7a, b [15]. Translational accuracy, defined as separation of two points $\Delta \mathrm{Ti}$, was measured at several points where displacement between one manipulated fragment (TFi) and the femur (TRi reference) was perceived to be greatest. For each fragment at least 6 data points were taken from the fluoroscopic images to determine the translational error (Fig. 7a). Rotational accuracy was measured as the difference between the axis defined by the femur $\vartheta R i$ (reference) and the axis defined by the fragment $\vartheta F i$. Translational and rotational accuracies were measured for each fragment in both coronal and sagittal planes (Fig. 7b). Experimental results are reported in Table I.

\section{Discussion}

The aim of this work is to make the RAFS system applicable and advantageous for fracture surgery. To demonstrate the performance of the new RAFS system, it was tested throughout laboratory trials and cadaveric trials. The experiments demonstrated that the system is able to perform 2 -fragments distal femur fracture surgeries. The metrics chosen for the system reduction accuracy evaluation, i.e. RMSE and MAE, give an account of how far the manipulated fragments are from the reduced position, and therefore, of the physical reduction procedure. On these metrics the RAFS system showed values close to the clinical acceptable ones $\left(1 \mathrm{~mm}, 5^{\circ}\right)$ on both laboratory models and cadaveric specimens. The best result in laboratory trials was obtained reducing a Y-shape 33-C1 complete articular fracture, with a residual reduction error (RMSE) of $0.73 \pm 0.1 \mathrm{~mm} / 3.2 \pm 0.2^{\circ}$ (fragment 2), and $1.44 \pm 0.1 \mathrm{~mm} / 2.47$ $\pm 0.3^{\circ}$ (fragment 1 ). Similar results were obtained in cadaveric trials. The RAFS system reduced a Tshape 33-C1 complete articular fracture with a residual reduction error of $1.41 \pm 0.3 \mathrm{~mm} / 3.12 \pm 0.4^{\circ}$ (fragment 1) and $0.93 \pm 0.2 \mathrm{~mm} / 3.3 \pm 0.5^{\circ}$ (fragment 2). Measured MAEs (in both lab and cadaveric trials) corroborate the findings above, showing that the system permits good reduction accuracies avoiding large deviations from the desired reduction. Slightly higher residual translations errors (RMSE and MAE) were found after the reduction of Y-shape 33-C1 in cadaveric trials ( $1.83 \pm 0.1 \mathrm{~mm} \mathrm{RMSE}, 2$ $\mathrm{mm}$ MAE), and T-shape 33-C1 in lab trials (1.73 $\pm 0.1 \mathrm{~mm}$ RMSE, $1.8 \mathrm{~mm}$ MAE). This looks related with the higher load applied by the RFMs during the physical reduction, i.e. $113.1 \pm 5.4 \mathrm{~N} / 3.2 \pm 0.3 \mathrm{Nm}$ (Cadaver Y-shape 33-Cl) and $112.6 \pm 15 \mathrm{~N} / 4.9 \pm 0.3 \mathrm{Nm}$ (Lab Model T-shape 33-Cl). These higher values of forces and torques depends on the soft tissues counteracting the reduction [11], which cause the pins to bend a little and increase the error. With this regard, Cadaver Y-shape 33-C1 presented a better developed musculature with respect to Cadaver T-shape $33-\mathrm{Cl}$, which might be related with the age of the specimens (Cadaver Y-shape 33-C1 was 73 years old, Cadaver T-shape 33-C1 was 103) and their soft tissues quality. Similarly, Lab Model T-shape 33-C1 was designed to have shorter ligaments (i.e. polypropylene bands, see Section VI) and, therefore, a stiffer knee joint with respect to Lab Model Yshape 33-C. The ER supported the fragment manipulation and facilitated the reduction of the fractures. Measured applied traction (ER) data further corroborate the above findings. This explains the slightly lower reduction accuracy-on average-of the new RAFS system with respect to the earlier prototype $\left(1.15 \mathrm{~mm}\right.$ and $\left.1.3^{\circ}\right)$ [9]. The correlation between applied loads and achieved reduction accuracy should be further investigated through extensive cadaveric trials including specimens with different ages, sex, and muscle mass. The surgical time length using the RAFS system was measured during cadaveric trials and resulted in about 150 minutes to reduce and temporarily fixate one fracture. Currently, the standard open procedure might last up to 100 minutes [16]. With respect to the clinical workflow, only the intra-operative procedure was considered, since it directly affects the 
patient (e.g. duration of the anesthesia, risk of infection) and the operating theatre management (e.g. operating theatre occupancy rate). The intra-operative procedure includes: pins insertion ( $35 \mathrm{~min}$ ), $2 \mathrm{D} / 3 \mathrm{D}$ registration ( $\sim 83 \mathrm{~min}$ ), robot setup ( $\sim 25 \mathrm{~min}$ ), and physical reduction and temporary fixation ( $\sim 8 \mathrm{~min}$ ). The $2 \mathrm{D} / 3 \mathrm{D}$ registration is a key part of the clinical workflow, enabling the image-guidance. However, at the current state of development, it is overly time consuming. Manual user interaction is required to identify the location of anatomical landmarks and surfaces to carry out the registration, making it time consuming and subjective. Automating the intraoperative 2D/3D registration-e.g. an automated 2D/3D registration framework for image-guided orthopedic surgery is proposed in [17]will potentially reduce the whole surgical time, while maintaining the benefits of the image-guided robotic assistance with respect to the standard open procedure, i.e. higher reduction accuracy, realtime 3D intraoperative imaging, minimum soft tissue damage, possibility of surgical pre-planning. Therefore, this aspect needs further attention in future development of the RAFS system.

Further studies are planned in the optimization of the 2D/3D registration framework for the RAFS system through the full automation of the procedure. Extensive cadaveric trials are also planned to gather more objective measurement of the system in the surgical environment. Preliminary hazard analysis for the RAFS system has been conducted [18], and extensive safety analysis is currently under investigation paving the way for clinical trials.

\section{Appendix}

The forward kinematics of the ER is based on the parameters shown in Fig. 8: $\theta$ l is the rotation of the entire ER around the axis perpendicular to the limb, $\mathrm{d} 2$ is the linear motion perpendicular to the limb axis, $\mathrm{d} 33$ is the linear motion for traction, and $\theta 4$ is the rotation around the axis of the limb. The desired target position of the foot reference frame RFF $(d e s)$ in respect to $\{0\}$ given by the vector:

$\mathrm{OP}_{\text {des }}=\left[\mathrm{X}_{\mathrm{des}} Y_{\operatorname{des}} Z_{\mathrm{des}} \theta_{\mathrm{Xdes}} \theta_{\mathrm{Ydes}} \theta_{\mathrm{Zdes}}\right]$

where $\mathrm{Xdes} Y$ desZdes are the Cartesian coordinates and $\theta \mathrm{xdes} \theta \mathrm{Ycles} \theta z d e s$ the Euler angles in respect to $\{0\}$. 
Figures

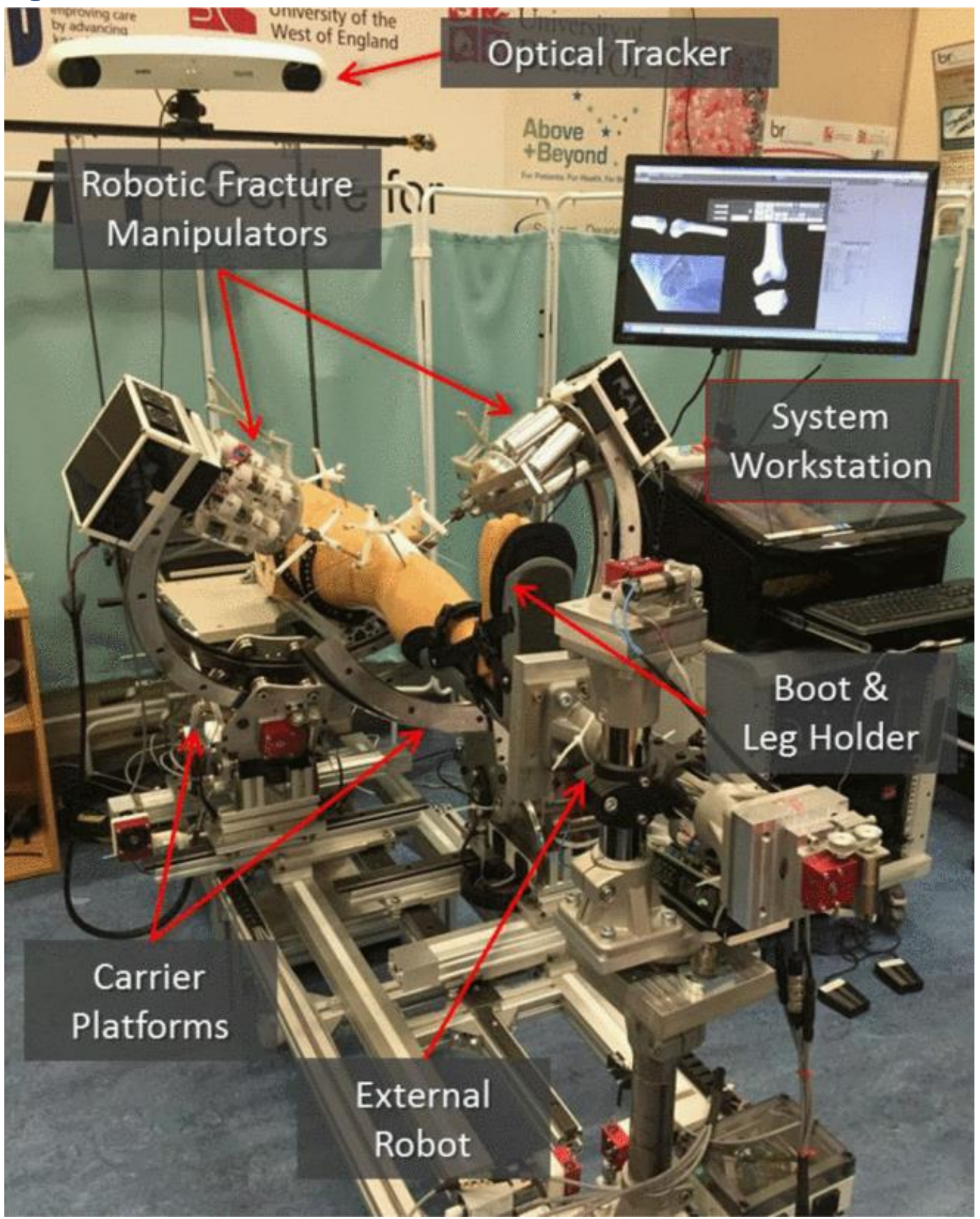

Fig. 1: RAFS surgical system 


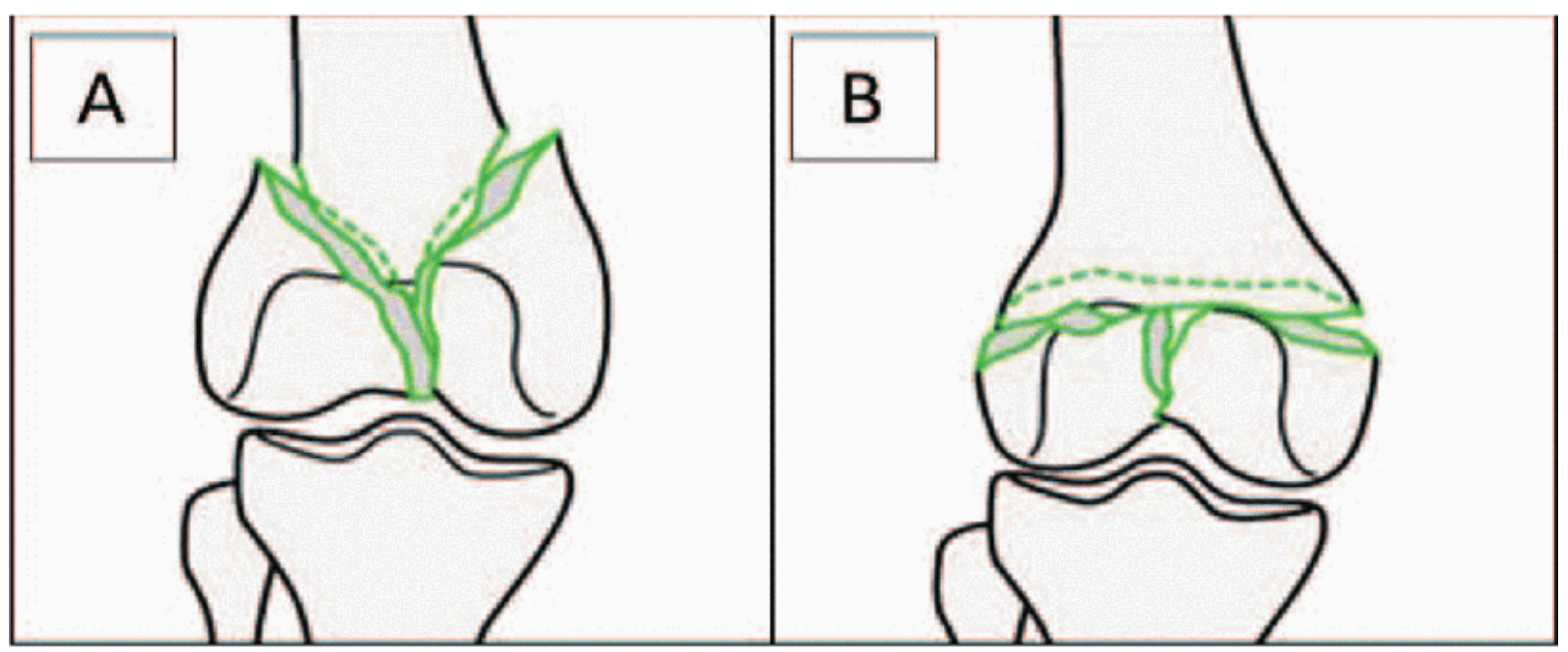

Fig. 2: Complete articular distal femur fractures: $\mathrm{Y}$-shape 33-C1 (a) and T-shape 33-C1 (b) used for RAFS system development and testing [3].

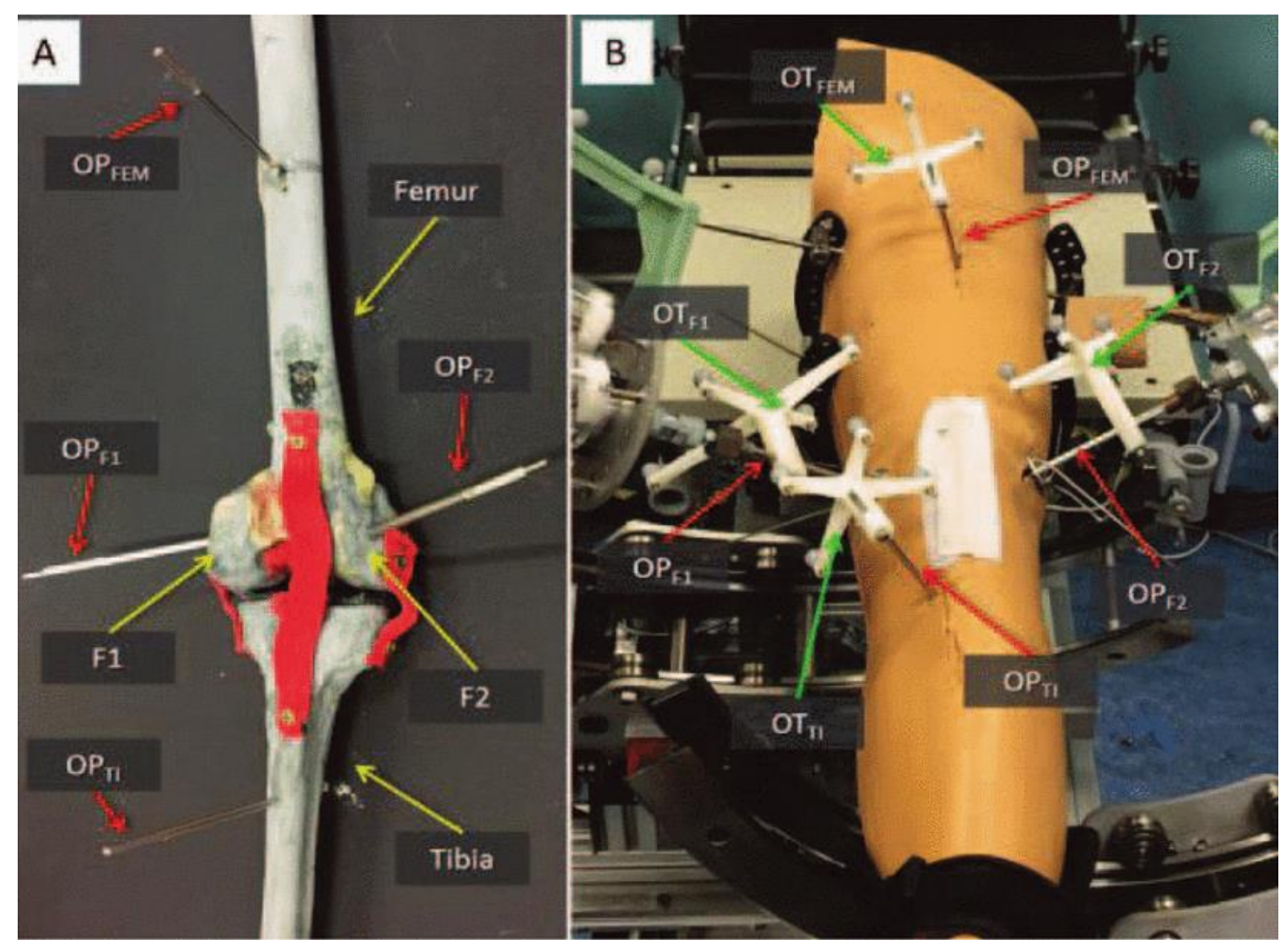

Fig. 3: Fractured femur model with orthopedic pins inserted (a); optical tools are inserted in a unique way into the tool sections of the pins allowing for intra-operative real-time imaging (b). 


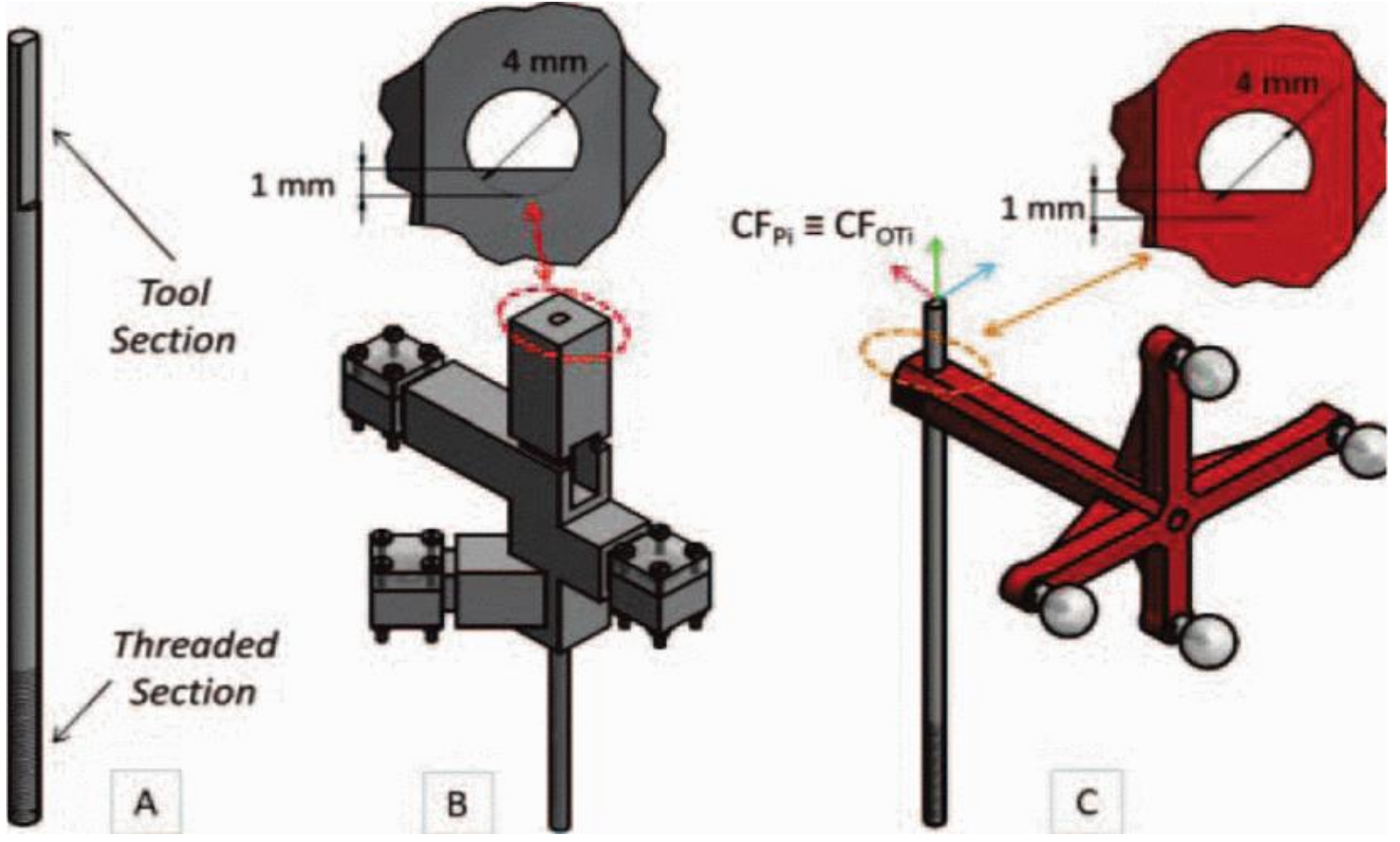

Fig. 4: Orthopedic manipulation pin: Tool section one-flat unique geometry (a). Registration tool (b) and optical tool (c) placed on the tool section of the pin. 


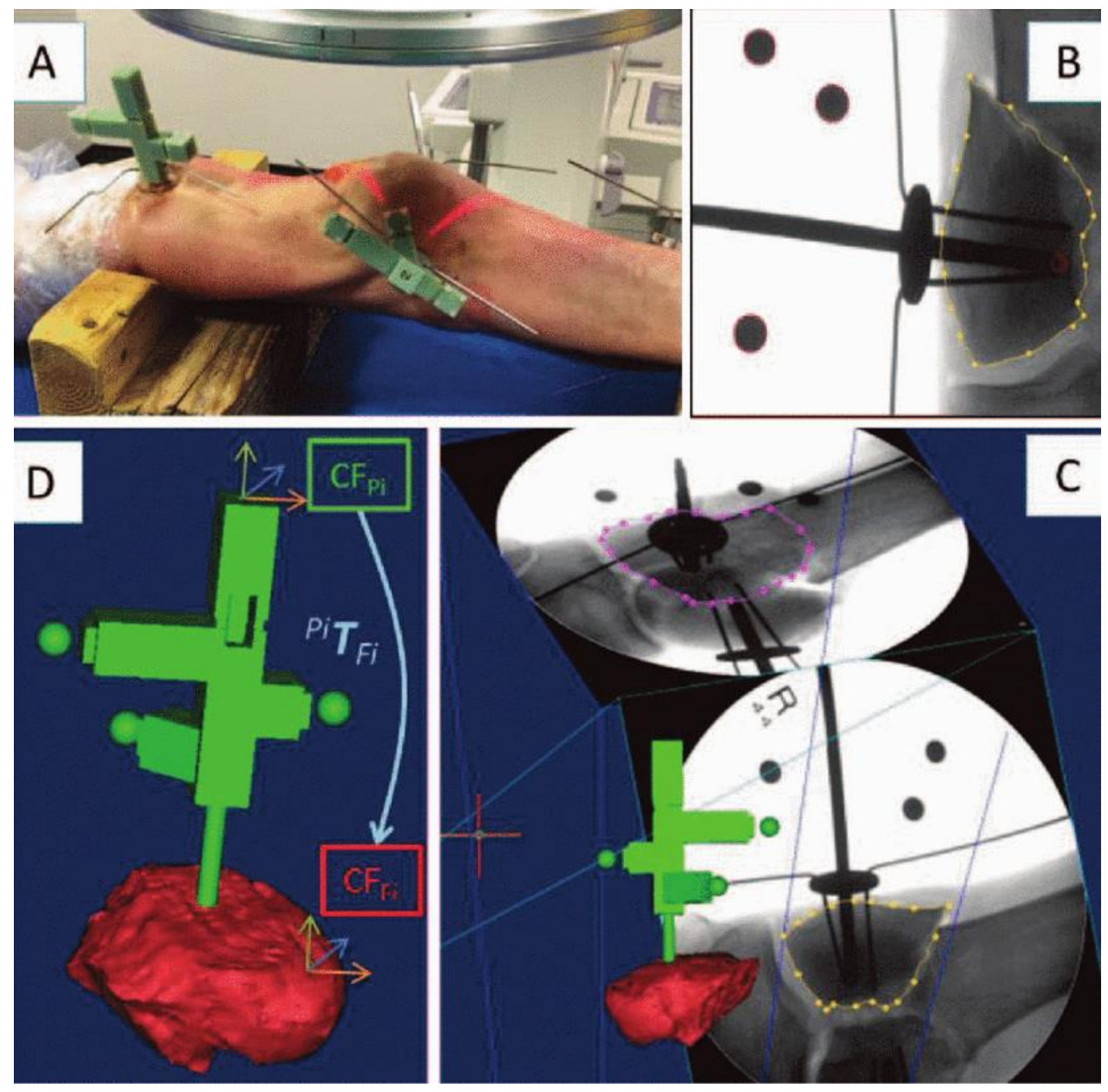

Fig. 5: Intra operative 2D/3D registration. Cadaveric specimen with registration tools inserted into orthopedic pins (a); intra-operative fluoroscopic image on coronal plane (b) of a bone fragment with orthopedic pin and registration tool; registration framework (c); bone fragment and pin models registered (d): The relative pose between the coordinate frames of the pin ( $\left.c f_{p i}\right)$ and the bone fragment $\left(\mathrm{cf}_{\mathrm{Fi}}\right)$ is known and defined by the homogeneous transformation PiTFi. 


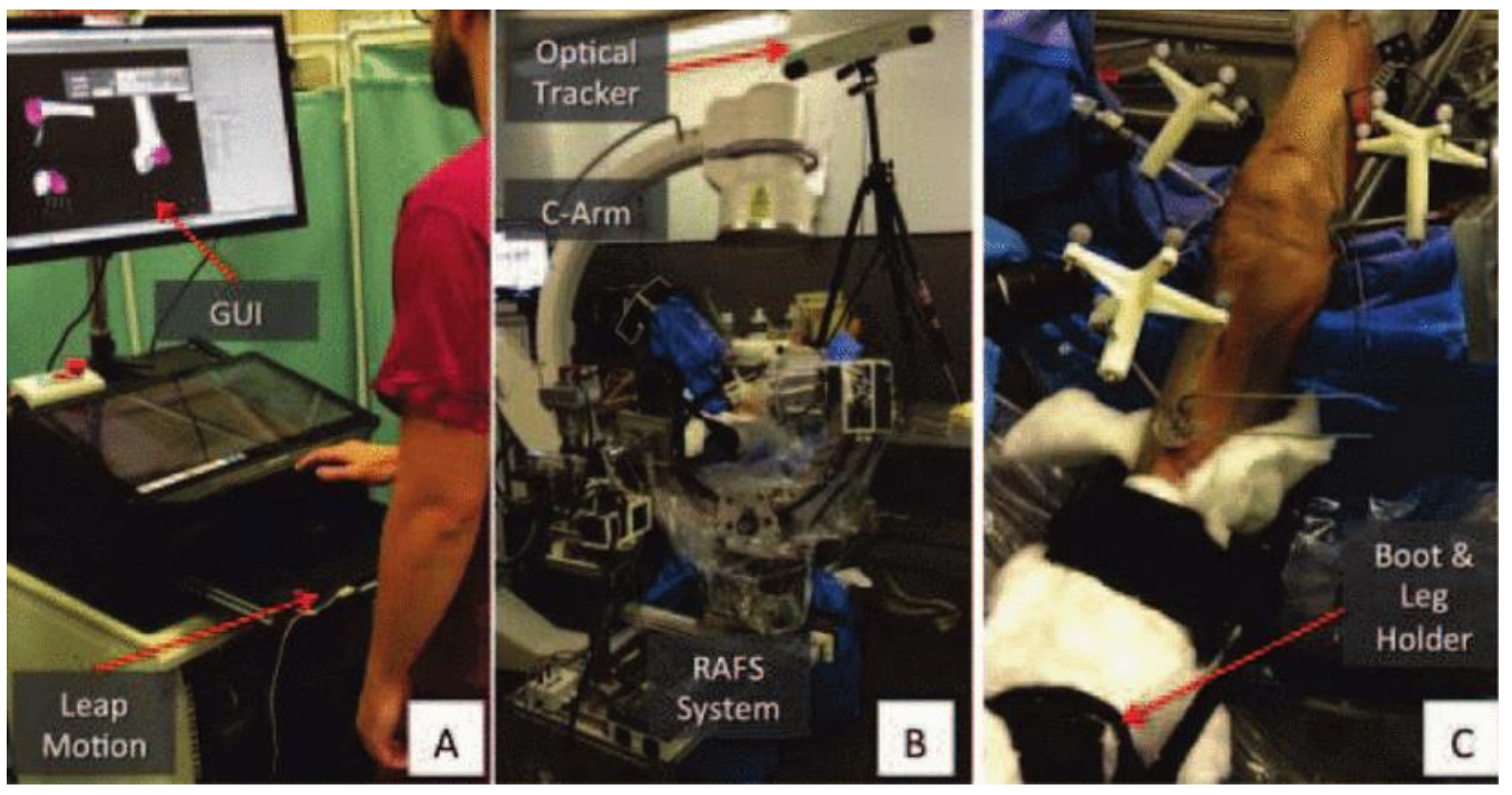

Fig. 6: RAFS system in cadaveric trials: Pre-operative virtual reduction of the fracture using the GUI (a); the RAFS system in the operating theatre (b) connected to a cadaveric specimen (c).

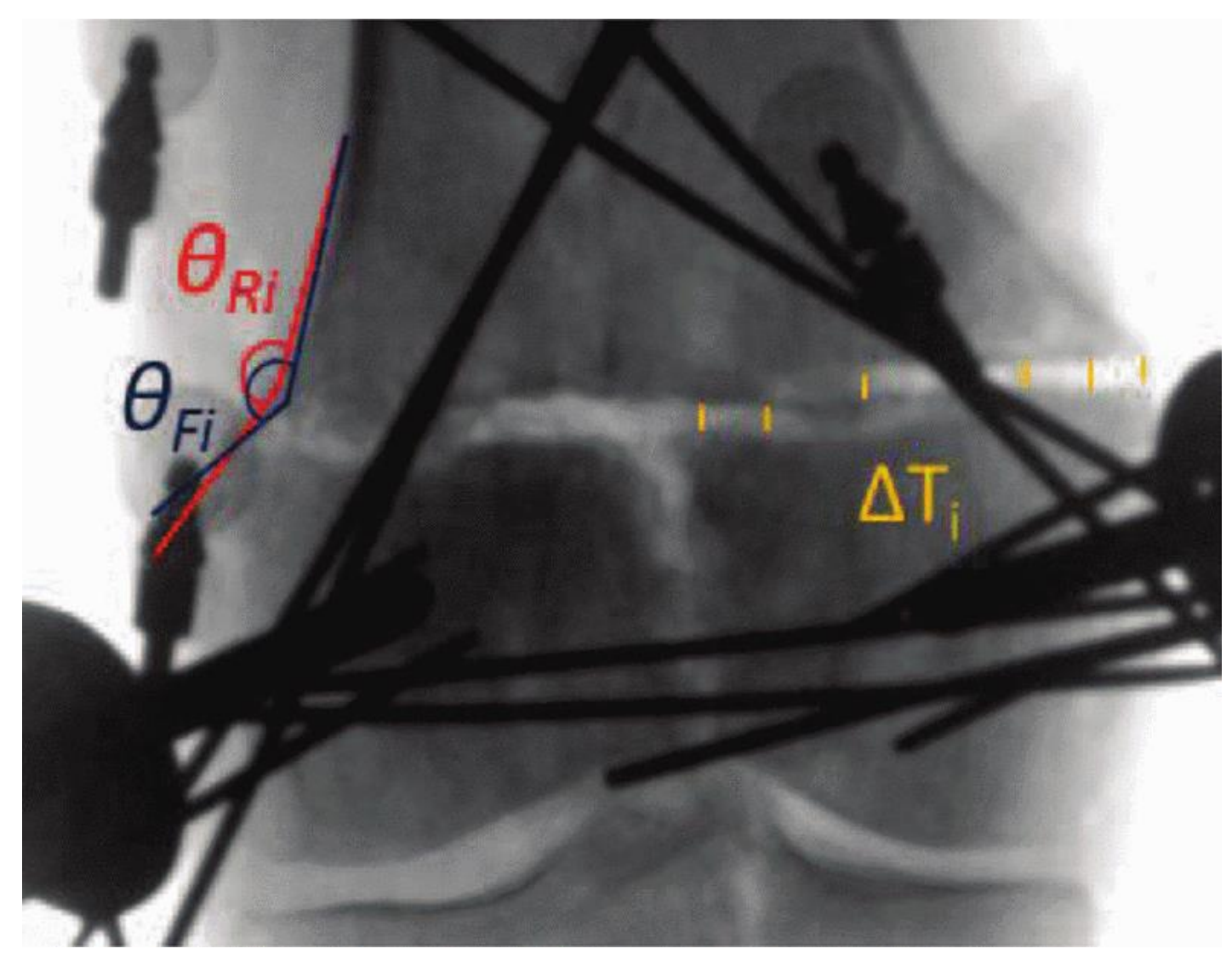

Fig. 7: Cadaveric trials results: Fluoroscopic image of a reduced T-shape 33-c1 fracture on the coronal and plane, with superimposed translational (orange) and rotational (red, blue) evaluation metrics. 


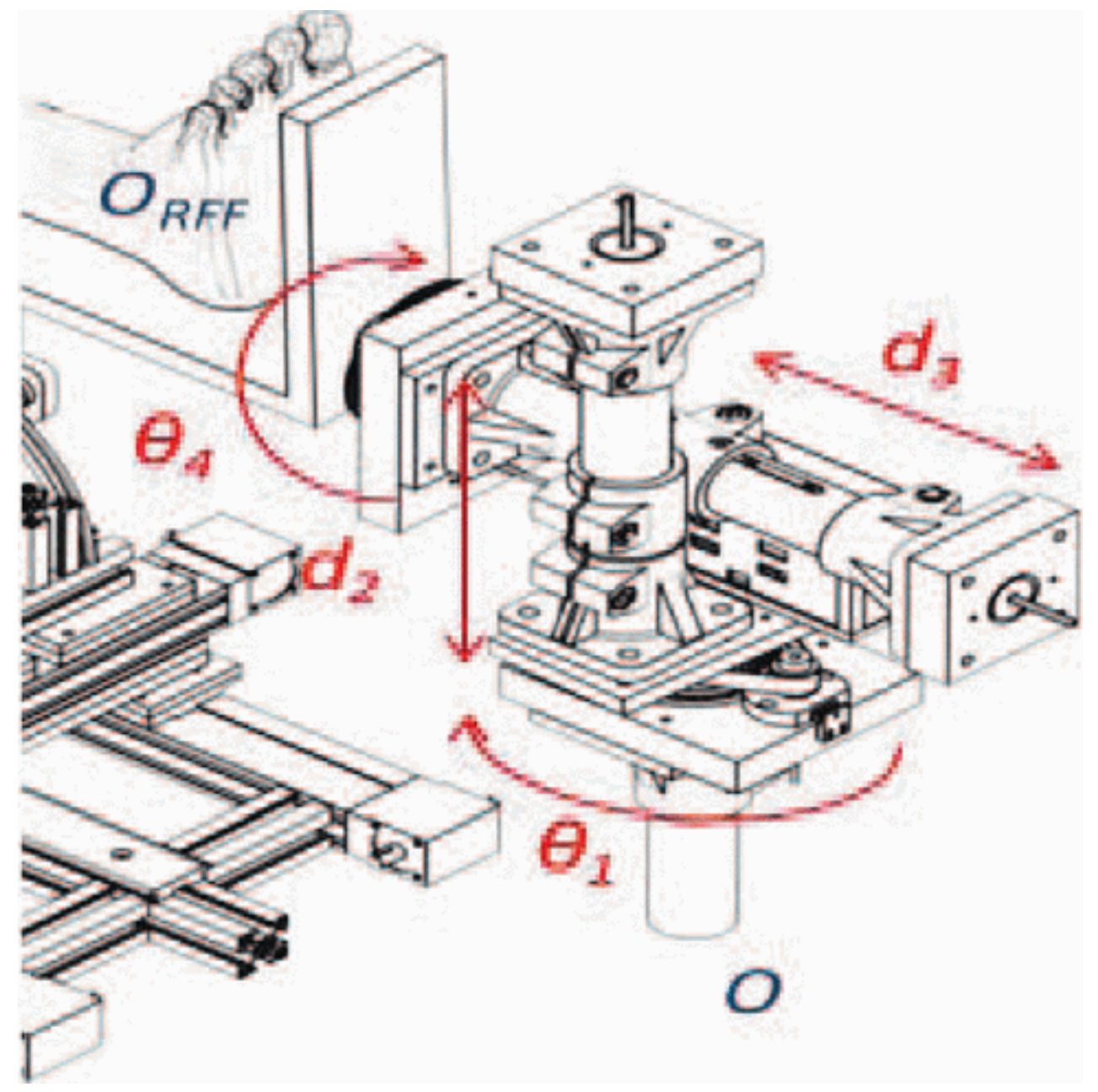

Fig. 8: External robot rotational $(\vartheta 1, \vartheta 4)$ and prismatic $\left(d_{2}, d\right)$ dof.

Table

\begin{tabular}{|c|c|c|c|c|c|c|c|}
\hline Fracture Type & $\begin{array}{l}\text { F1 } \\
\text { RMSE }\end{array}$ & $\begin{array}{l}\text { F1 } \\
\text { MAE }\end{array}$ & $\begin{array}{l}\text { F2 } \\
\text { RMSE }\end{array}$ & $\begin{array}{l}\text { F2 } \\
\text { MAE }\end{array}$ & $\begin{array}{l}\text { Applied Traction } \\
\text { (ER) }\end{array}$ & $\begin{array}{l}\text { Applied Load } \\
\text { (RFMs) }\end{array}$ & Surgery Time \\
\hline $\begin{array}{l}\text { Lab Model }{ }^{2} \\
\text { Y-Shape 33-Cl }\end{array}$ & $\begin{array}{l}1.44 \pm 0.1 \mathrm{~mm} \\
2.47 \pm 0.3^{\circ}\end{array}$ & $\begin{array}{l}1.5 \mathrm{~mm} \\
3.5^{\circ}\end{array}$ & $\begin{array}{l}0.73 \pm 0.1 \mathrm{~mm} \\
3.2 \pm 0.2^{\circ}\end{array}$ & $\begin{array}{l}0.9 \mathrm{~mm} \\
3.4^{\circ}\end{array}$ & $27.5 \pm 3.1 \mathrm{~N}$ & $\begin{array}{l}44.9 \pm 1.2 \mathrm{~N} \\
3.6 \pm 0.2 \mathrm{Nm}\end{array}$ & n.a. \\
\hline $\begin{array}{l}\text { Lab Model" } \\
\text { T-Shape 33-Cl }\end{array}$ & $\begin{array}{l}1.42 \pm 0.3 \mathrm{~mm} \\
3.41 \pm 0.4^{\circ}\end{array}$ & $\begin{array}{l}1.5 \mathrm{~mm} \\
3.8^{\circ}\end{array}$ & $\begin{array}{l}1.73 \pm 0.1 \mathrm{~mm} \\
1.41 \pm 0.3^{\circ}\end{array}$ & $\begin{array}{l}1.8 \mathrm{~mm} \\
1.8^{\circ}\end{array}$ & $37.1 \pm 0.8 \mathrm{~N}$ & $\begin{array}{l}112.6 \pm 15 \mathrm{~N} \\
4.9 \pm 0.3 \mathrm{Nm}\end{array}$ & n.a. \\
\hline $\begin{array}{l}\text { Cadaver } \\
\text { Y-Shape 33-Cl }\end{array}$ & $\begin{array}{l}1.83 \pm 0.1 \mathrm{~mm} \\
2.4 \pm 0.3^{\circ} \mathrm{s}\end{array}$ & $\begin{array}{l}2 m m \\
2.9^{\circ}\end{array}$ & $\begin{array}{l}0.85 \pm 0.3 \mathrm{~mm} . \\
2.2 \pm 0.1^{\circ} \mathrm{s}\end{array}$ & $\begin{array}{l}1.25 \mathrm{~mm} \\
2.4^{\circ}\end{array}$ & $51.4 \pm 2.8 \mathrm{~N}$ & $\begin{array}{l}113.1 \pm 5.4 \mathrm{~N} \\
3.2 \pm 0.3 \mathrm{Nm}\end{array}$ & $154 \min$ \\
\hline $\begin{array}{l}\text { Cadaver } \\
T \text {-Shape 33-Cl }\end{array}$ & $\begin{array}{l}1.41 \pm 0.3 \mathrm{~mm}, \\
3.12 \pm 0.4{ }^{\circ} \mathrm{s}\end{array}$ & $\begin{array}{l}1.75 \mathrm{~mm} \\
3.2^{\circ}\end{array}$ & $\begin{array}{l}0.93 \pm 0.2 \mathrm{~mm}, \\
3.3 \pm 0.5^{\circ} \mathrm{s}\end{array}$ & $\begin{array}{l}1.25 \mathrm{~mm} \\
3.4^{\circ}\end{array}$ & $10.8 \pm 2.3 \mathrm{~N}$ & $\begin{array}{l}69.9 \pm 4.4 \mathrm{~N} \\
4.8 \pm 0.4 \mathrm{Nm}\end{array}$ & $148 \min$ \\
\hline
\end{tabular}

Table I: Results: Physical reduction with RAFS system. 